\title{
SIRT1 Gene Single Nucleotide Polymorphisms and Female Infertility: A Cross Sectional Study In Pakistan
}

\author{
Faiza Alam ${ }^{1}$, Rehana Rehman ${ }^{2}$, Syeda Sadia Fatima ${ }^{2}$, Mussarat Ashraf ${ }^{2}$, and Taseer \\ Ahmed Khan ${ }^{1}$ \\ ${ }^{1}$ University of Karachi \\ ${ }^{2}$ Aga Khan University
}

May 6, 2020

\begin{abstract}
Aim \& Objective: Silent information Regulators (SIRT1) gene stimulates the expression of antioxidants and repairs damaged cells. It affects the mitochondrial activity within the oocytes to overcome the oxidant stress. We aimed to assess an association of SIRT1 polymorphism (Tag SNPs rs10509291 and rs12778366) with fertility, and assess serum levels of follicle stimulating hormone (FSH), luteinizing hormone (LH), estradiol, progesterone, manganese superoxide (MnSOD) and SIRT1. Material and Methods: In this cross-sectional study, 207 fertile and 135 infertile subjects between the ages of 18-45 year were recruited. Polymerase chain reaction (PCR) was performed; products were electrophoresed in a $2 \%$ agarose gel. Descriptive analysis of continuous variables was expressed as mean \pm standard deviation. Mann-Whitney test was performed for comparison of groups, p value $<0.001$ was considered significant. Single Nucleotide Polymorphism (SNP) data was analyzed by applying chi-squared statistics. Results: All subjects were age matched $(\mathrm{p}=0.896)$. SIRT1 levels were significantly lower in infertile females when compared with fertile subjects $(\mathrm{p}<0.001)$. AA (rs10509291) and CC (rs12778366) variant frequency was higher in the infertile than fertile subjects $(\mathrm{p}<0.01)$. Similarly, the frequency of A allele (rs10509291) and C allele (rs12778366) was higher in infertile subjects $(\mathrm{p}<0.001)$. Infertile females $(29 \%)$ showed existence of SNP rs10509291 while 49\% demonstrated genetic variation of rs12778366. MnSOD and SIRT1 levels were found to be lower in these subjects. Conclusion: Presence of SIRT1 genetic variants (rs10509291 and rs12778366) apparently disturbs the expression of SIRT1 deteriorating mitochondrial antioxidant function within the oocytes, instigating oxidative stress within. Their probable effect on modulating oocyte maturation may be the cause of infertility in females.
\end{abstract}

\section{What's already known about this topic?}

Genetic mutations are responsible for causing oxidative stress giving rise to infertility.

\section{What does this article add?}

Genetic mutations of SIRT1 causes oxidative stress, which could be a possible answer to unexplained infertility.

Keywords: Female infertility, Infertility, MnSOD, Single Nucleotide Polymorphism, SIRT1.

\section{Introduction:}

Infertility is a multifactorial syndrome where a couple fails to conceive even after 12 months of regular unprotected sexual interaction [1]. It's a growing global burden effecting $23 \%$ of married couples in reproductive age bracket in Pakistan and increasing at rate of $4 \%$ annually [1]. There are various known causes 
responsible for this increasing burden ranging from reproductive disorders, endocrine imbalance, obesity and hypothyroidism to psychological disturbance [2].

The fertilization of the ovum has a reliance on the quality of ovum, which is governed by the internal environment by various factors. Oxidative stress (OS) stands a justified cause of oocyte immaturity [3]. The chromosomal segregation within an oocyte, fertilization failure of an ovum and fragmentation of the embryo depends majorly on the redox imbalance, caused by the mitochondrial dysfunction $[4,5]$. Redox activity has been reported to get regulated by the enzymatic activities of hormones and protein.

Silent information Regulators (SIRT) (1-7) is recognized as a regulatory protein affecting the oxidative milieu of the reproductive system [6]. Out of the 7 classes, SIRT1 serves as a "rescue gene," directly deacetylating transcription factor carrying out the cellular stress response [7]. The gene incites the antioxidants expression, which aids in repairing the damaged cells due to oxidative disturbances within the cells and also prevents dysfunction of the cells [8]. Very little evidence is available but some human studies indicate the presence of SIRT1 signaling in the ovarian cells' response to the insulin sensitizer drugs like metformin (MetF), which is well recognized to enhance the $\mathrm{NAD} \pm / \mathrm{NADH}$ ratio and SIRT1 both, suggesting its role in balancing the redox activity as an outcome [9]. This implies the role of SIRT1 in controlling the continuing OS, perhaps by up-regulating the key mitochondrial antioxidant enzymes i.e. catalase and manganese/ mitochondrial superoxide dismutase (MnSOD) [8]. SIRT1 genetic crosstalk has been associated with altered regulation of MnSOD [10]. Disturbed levels of LH have been reported with raised OS parameters rendering this to be a probable cause multiple abortions and infertility in women [10]. Increased $\mathrm{H}_{2} \mathrm{O}_{2}$ causes aging of the cells as shown in the endothelial cells and SIRT1 activation/induction protects against the damaging effects of hydrogen peroxide [11]. Moreover, significant change of SIRT1 mRNA has also been observed with increase in reactive oxidative species $[6,12]$. The raised levels of OS markers are hypothesized to compromise the nuclear maturation and the mitotic spindles of the maturing oocytes. The SIRT1 gene spreads over 33.72 $\mathrm{kb}$, on chromosome 10q21.3 and is encoded in nine exons intermittently arranged along with eight introns. SIRT1 null offspring mice had smaller birth size and most of them died through their early postnatal period. Studies in mice have indicated that SIRT1 gene plays a key role in regulating metabolic and mitochondrial functions in various tissues through certain targets including PPAR $\gamma$, PGC-1 $\alpha$, and AMP kinase [13 - 15].

Genetic discrepancies have also been established as the solid grounds in around $5-10 \%$ of infertile females [16]. Since SIRT1 is capable of moderating the key target proteins maintaining mitochondrial function [17, 18], it is expected that SIRT1 polymorphism (Tag SNPs rs10509291 and rs12778366) in females can cause a decrease in antioxidants, which may disturb the microenvironment hence causing infertility. Studies focusing on SIRT1 genetics associated with human infertility are relatively very few as compared to data available from animal studies. Some studies have identified an association ofSIRT1 genes with predisposition of T2DM and obesity [19], which are common attributes of infertile females, but so far no study up to our knowledge indicates the association of SIRT1 polymorphism with infertility in females.

As a known fact, cyclic changes of FSH and LH are essential for normal follicular maturation, yet in normal conditions low or resting LH concentrations are also ample to cause required maturation of follicles and oocytes. Factor like SIRT1 polymorphism for changes in MnSOD levels may have adverse effects. Thus, we aimed to estimate the frequency of SIRT1 polymorphism (Tag SNPs rs10509291 and rs12778366) in fertile and infertile females, along with the change in the serum levels of follicle stimulating hormone (FSH), luteinizing hormone (LH), estradiol and manganese/mitochondrial superoxide dismutase (MnSOD) in association with the genotype variants.

\section{Material \& Methods:}

This pilot study was designed as cross-sectional study on three hundred and forty two females was conducted at Aga Khan University, Karachi while the recruitment was done at "Australian Concept Infertility Medical Centre, Karachi (ACIMC)" during the period of October 2017 till December 2018. After approval from Aga Khan Institutional ethical committee (4956-BBS-ERC), the research was conducted in accordance with the guidelines of Helenski and all the participants signed a written consent. Online software at Open Source 
Epidemiologic Statistics for Public Health was used to calculate the sample size [20]. In order to achieve $80 \%$ power with an odds ratio of minimum 2, hypothetical proportion of fertile with exposure was assumed to be $22 \%$, with prevalence of infertility $23 \%$ [1] and two-sided confidence (1-alpha) of $5 \%$ was observed. The sample size was calculated to be 342 females [20]. All age females between the ages of 18-45 years with a child less than 2 years of age from all ethnic groups were recruited as fertile. Recruitment for infertile females was on the basis of the indicated/diagnosis made by a physician according to the criteria of infertility (failure to conceive since 2 years despite continuous attempts), between the ages of $18-45$ years, from all ethnic backgrounds.

Infertile females due to male factor causes or due to tubal blockade were excluded. In addition females with history of artificial reproductive techniques (ART) in previous pregnancies, recurrent miscarriages, thyroid dysfunctions, gynaecological tumours, hypertension and diabetes were not included. Those with serious general health status, using oral contraceptive pills and hormonal treatments or following any contraceptive measures were also disqualified for enrolment. Body mass index (BMI) of all patients was calculated by following formula: BMI $=$ weight $(\mathrm{Kg}) /$ height $\left(\mathrm{m}^{2}\right)[21]$.

Biochemical Estimation:

Ten-milliliter of venous blood was collected from the ante-cubital vein of all the study subjects. Serum was extracted by centrifugation of whole blood and was stored for analysis of estradiol (Kit Cat. No. 07BC-1111 by MP BioCheck, Inc), follicular stimulation hormone (Kit Cat\# DKO010 by Diametra Immuno Assays S.A. Belgium), leutinizing hormone (Kit Cat. No DKO009 by Diametra source ImmunoAssays S.A), SIRT1 (Kit Cat. No. SG-10458 by Sino Gene Clone Biotech Co., Ltd) and manganese superoxide dismutase using commercially prepared Elisa kits (Kit Cat. No.SG-10731 by Sino Gene Clone Biotech Co., Ltd).

\section{Genetic Evaluation:}

Whole blood sample was used to extract DNA using commercially existing DNA extraction kit from Promega Genomic (Cat \# A1125). Primers were designed using University of California Santa Cruz (UCS) genome browser primer blast tool for both the SNPs of SIRT1 [rs10509291 (Forward TTCCAACTACGCTATCAATCT; Reverse CAGATAGAAGCCAAGGGTGT) and SIRT1 rs12778366 (Forward TAAGGCTTCTAGGACTGGAG; Reverse CTAAGGTCCTATCTACATCC)]. These primers were used for gene amplification. Product size of 372 base pairs for rs10509291 and 911 base pairs for rs12778366 were produced. PCR was performed in $0.2 \mathrm{ml}$ tube containing $50 \mathrm{ul}$ total reaction mixture. Genotype quality control was performed in $10 \%$ of the samples by duplicate checking (rate of concordance in duplicates was $>99 \%$ ). Further reconfirmation of genotyping accuracy, was made by sequencing several samples with detected polymorphic variants (Macrogen, Korea) (Figure 1). Data storage and analysis was done using SPSS version 21. Descriptive analysis of continuous variables was expressed as mean \pm standard deviation. Mann-Whitney test was performed to compare the groups and $\mathrm{p}$ value $<0.001$ was considered significant. HWE was calculated for single nucleotide polymorphisms (SNP) data. SNP data was analyzed for genotype and allele frequency determination by applying chi-squared statistics.

\section{Results:}

All subjects were age matched and therefore no difference was observed in between the two groups $(\mathrm{p}=0.896)$.

\section{Biochemical:}

With a mean BMI of $24.75 \pm 2.6 \mathrm{~kg} / \mathrm{m}^{2}(\mathrm{p}=0.021)$, Progesterone and estradiol levels were lower in infertile females $(\mathrm{p}=0.041 ; \mathrm{p}<0.001$, respectively). Furthermore, LH $(\mathrm{p}<0.001)$, MnSOD $(\mathrm{p}<0.001)$ and SIRT1 $(\mathrm{p}<0.001)$ levels were also noted to be significantly lower in infertile when compared with the fertile subjects. FSH showed no difference between the groups. (Table 1)

\section{Genetic:}

Genotype for SIRT1 rs12778366 and rs10509291 polymorphism was performed, where controls had HardyWeinberg's equilibrium (HWE) $>0.05$. Significant difference was noticed between fertile and infertile subjects 
when frequencies of both SNP were compared $(\mathrm{p}<0.01)$. (Table 2).

Odds ratios of allele frequency was calculated for both the SNPs. Fertile subjects demonstrated a higher frequency of $\mathrm{T}$ allele in both SNPs however, the A allele of rs10509291 and $\mathrm{C}$ allele of rs12778366 was higher in infertile subjects $(\mathrm{p}<0.001, \mathrm{p}<0.001)$. (Table 3$)$

When stratifying on the basis of existence of the genetic variant, infertile females exhibited significant higher percentage as compared to the fertile females, $29 \%$ of infertile females showed existence of SNP rs10509291 and $49 \%$ showed rs12778366 positivity. (Table 4 )

Table 5 expresses significance in the serum levels of hormones in the overall subjects $(n=342)$ between the females having and not having the respective mutation. A similar trend of LH, MnSOD and SIRT1 was noticed in both the variants, the serum levels were significantly low in subjects having the mutations $(\mathrm{CC} / \mathrm{CT}$ or $\mathrm{AA} / \mathrm{AT})$ when compared with the ones not having it (TT). However, progesterone and FSH demonstrated no significant difference. (Table 5 a \& b)

\section{Discussion:}

MnSOD has a significant role in the aerobic respiration of cells but also has a crucial role in opposing oxidative stress (OS) with in a cell [22]. The precise mechanism although is not clear yet post-translational, post-transcriptional modification are thought to counter the effects of OS [23]. SIRT1 activity has recently been suggested to inhibit oxidant effect through use of dietary antioxidant methods by its effect on mitochondrial antioxidant system [24]. Our study is in line with these results, demonstrating a low level of MnSOD advocating oxidant environment in infertile females owing to a decrease antioxidant control. The damaging effect of MnSOD on SIRT1 activity has been reported [25] whereas MnSOD is a mitochondrial antioxidant, which balances $\left(\mathrm{O}^{2-}\right)$ and forms $\mathrm{H}_{2} \mathrm{O}$ in the mitochondrial matrix [26].

We observed a minor allele frequency (MAF) of both Tag SNPS rs10509291and rs12778366 respectively in agreement to previous genome project [27]. This change is also known to affect the SIRT in infertile females; which causes impaired oocyte maturation due to increase in OS in granulosa cells hence leading to infertility $[28,29,30]$. We have observed lower level of antioxidant MnSOD in infertile subjects with SIRT1 polymorphism and disturbedSIRT1 genetic signaling which is similar to previous studies [25,10]. Similarly role of SIRT1 in moderating atypical meiotic spindle has been identified in mouse model [31].SIRT1 rs12778366 has been reported in patients with pituitary adenoma like gonadotropinomas [32]. This may account for the hormonal (FSH and LH) imbalances seen in the infertile subjects having SIRT1 polymorphisms. Both FSH and LH are required for normal follicular estradiol biosynthesis [33]. In the current study we observed low levels of LH with low levels of estradiol in the infertile group of subjects $(\mathrm{p}<0.001)$, which could be a cause of infertility in females. However the underlying factor causing the disturbance in the levels of these reproductive hormones might be existence of OS within the granulosa cells in response to the constant emotional stress experienced by the infertile females, as illustrated/described in our previous study [3]. Another animal study shows that SIRT1-null female mice have unproductive oestrous cycles, hormonal disturbance, inadequate follicular maturation and improper embryo development [34]. We can thus hypothesize that SIRT1 gene polymorphism leads to development of OS, which can give rise to infertility probably due to its effect on oocyte microenvironment (Figure 2). This pilot study however is limited due to small sample size and exploration of few OS markers. Furthermore, the study does not mention about remedies to OS .The results although are preliminary and insufficient to draw an effective conclusion yet emphasizes the importance of genetic polymorphism as a causal mechanism for OS leading to infertility. This paves the pathway for validation of results with further exploration of genome-based studies. The extension of this study would be the study of these parameters in relation to the oocyte maturation and pregnancy outcomes in patients undergoing ART. Further exploration and correction can help in defining preventive measures for reproductive health. There is a need for large prospective studies to identify the causal links between these markers. Thus we conclude that SIRT1 genetic variants (rs10509291 and rs12778366) might be responsible for instigation of OS as is indicated by decrease in the levels of antioxidants (MnSOD and SIRT1). The induced OS is likely to impair microenvironment in the ovaries that has disturbed the hormonal balance and likely to impair oocyte 
maturity required for fertilization, implantation and hence conception.

We suggest that female subjects with suboptimal levels of antioxidants and low reproductive hormones are the ones who need thorough investigations and counselling before induction in ART. Furthermore, regular exercise and antioxidant diet plans could be recommended to such couples as a part of treatment plan. This will not only reduce the unnecessary burden on the cost of treatment but will also lessen the psychological stress due to repeated failures of ART. Here is the time where we suggest the role of geneticist to relate genetic polymorphism with OS markers and suggest options to the infertile couples.

\section{Acknowledgment:}

All authors have contributed significantly and all authors are in agreement with the content of the manuscript. All authors humbly recognize Pakistan Science Foundation (PSF) for the granting us funds [PSF/Res/S-AKU/Med(479)] to extend our work. Furthermore, we acknowledge Ms. Nida Farooqui for her valuable inputs.

\section{References:}

1 Sami N, Ali TS, Wasim S \& Saleem S. Risk factors for secondary infertility among women in Karachi, Pakistan. PLoS One 2012;7, e35828, doi:10.1371/journal.pone.0035828.

2 Arain F, Arif N \& Halepota H. Frequency and outcome of treatment in polycystic ovaries related infertility. Pak J Med Sci 2015;31, 694.

3. Alam F, Khan TA, Amjad S, Rehman, R. Association of oxidative stress with female infertility-A case control study. J Pak Med Assoc 2019;69.

4. Benkhalifa, M. et al. Mitochondria: participation to infertility as source of energy and cause of senescence. Int J Biochem Cell Biol 2014;55, 60-64.

5 Ishii, T. et al. Genetically induced oxidative stress in mice causes thrombocytosis, splenomegaly and placental angiodysplasia that leads to recurrent abortion. Redox Biol 2014;2, 679-685.

6 Ruder, E. H., Hartman, T. J., Reindollar, R. H. \& Goldman, M. B. Female dietary antioxidant intake and time to pregnancy among couples treated for unexplained infertility. Fertil Steril 2014;101, 759-766.

7 Brunet, A. et al. Stress-dependent regulation of FOXO transcription factors by the SIRT1 deacetylase. Science 2004;303, 2011-2015.

8 Caito, S. et al. SIRT1 is a redox-sensitive deacetylase that is post-translationally modified by oxidants and carbonyl stress. The FASEB Journal 2010;24, 3145-3159.

9 Reverchon, M. et al. Visfatin is expressed in human granulosa cells: regulation by metformin through AMPK/SIRT1 pathways and its role in steroidogenesis. Mol Hum Reprod 2013;19, 313-326.

10 Peck, B. et al. SIRT inhibitors induce cell death and p53 acetylation through targeting both SIRT1 and SIRT2. Molecular cancer therapeutics 2010;9, 844-855.

11 Lin, X.-L. et al. Inhibition of hydrogen peroxide-induced human umbilical vein endothelial cells aging by allicin depends on Sirtuin1 activation. Med Sci Mon: Int Med J Exp Clin Res 2017;23, 563.

12. $\mathrm{Xu}$, D. et al. miR-22 represses cancer progression by inducing cellular senescence. J Cell Biol 2011;193, 409-424.

13. Sack, M. N. \& Finkel, T. Mitochondrial metabolism, sirtuins, and aging. CSH Perspect Biol 2012;4, a013102.

14. Hoessli, D. C. et al. SIRT1: Importance of O-GlcNAc and Phosphorylation Interplay in Aging and Diabetes. Pak J Zool 2013;45, 27-34. 
15. Zhong, L. \& Mostoslavsky, R. Fine tuning our cellular factories: sirtuins in mitochondrial biology. Cell Met 2011;13, 621-626.

16. Fatima, S. S. et al. Polymorphism of the renalase gene in gestational diabetes mellitus. Endocrine 2017;55, 124-129.

17. Cantó, C. et al. AMPK regulates energy expenditure by modulating NAD+ metabolism and SIRT1 activity. Nature 2009;458, 1056.

18. Kume, S. et al. Calorie restriction enhances cell adaptation to hypoxia through Sirt1-dependent mitochondrial autophagy in mouse aged kidney. J Clin Invest 2010;120, 1043-1055.

19. Zillikens, M. C. et al. SIRT1 genetic variation is related to BMI and risk of obesity. Diabetes 2009;58, 2828-2834.

20 Kelsey, W. E., Kelsey, J. L., Whittemore, A. S., Evans, A. S. \& Thompson, W. D. Methods in observational epidemiology 26 Monographs in Epidemiology 1996.

21 Garrow, J. S. \& Webster, J. Quetelet's index (W/H2) as a measure of fatness. Int J Obes 1985;9, 147-153.

22 Zhao, Y. et al. p53 translocation to mitochondria precedes its nuclear translocation and targets mitochondrial oxidative defense protein-manganese superoxide dismutase. Cancer research 2005;65, 3745-3750.

23 Hussain, S. P. et al. p53-induced up-regulation of MnSOD and GPx but not catalase increases oxidative stress and apoptosis. Cancer research 2004;64, 2350-2356.

$24 \mathrm{Liu}, \mathrm{J}$. et al. Delay in oocyte aging in mice by the antioxidant N-acetyl-L-cysteine (NAC). Hum Reprod 2012;27, 1411-1420.

25 Solomon, J. M. et al. Inhibition of SIRT1 catalytic activity increases p53 acetylation but does not alter cell survival following DNA damage. Mol Cell Biol 2006;26, 28-38.

26 Ozden, O. et al. Acetylation of MnSOD directs enzymatic activity responding to cellular nutrient status or oxidative stress. Aging (Albany NY) 2011;3, 102-107.

27 Clarke, L. et al. The international Genome sample resource (IGSR): A worldwide collection of genome variation incorporating the 1000 Genomes Project data. NucleicAacids Res 2017;45, D854-d859, doi:10.1093/nar/gkw829.

28 North, B. J. \& Verdin, E. Sirtuins: Sir2-related NAD-dependent protein deacetylases. Genome Biol 2004;5, 224.

29 Tatone, C. et al. Sirtuin functions in female fertility: possible role in oxidative stress and aging. Oxid Med and Cell Longev 2015.

30 Yeung, F. et al. Modulation of NF- $x$ B-dependent transcription and cell survival by the SIRT1 deacetylase. The EMBO journal 2004;23, 2369-2380.

31 Di Emidio, G. et al. SIRT1 participates in the response to methylglyoxal-dependent glycative stress in mouse oocytes and ovary. BBA-Mol Basis Dis, 2019; doi:10.1016/j.bbadis.2019.02.011.

32 Glebauskiene, B. et al. Association of FGFR2 rs2981582, SIRT1 rs12778366, STAT3 rs744166 gene polymorphisms with pituitary adenoma. Oncology letters 2017;13, 3087-3099, doi:10.3892/ol.2017.5840.

33 Chappel, S. C. \& Howles, C. Reevaluation of the roles of luteinizing hormone and follicle-stimulating hormone in the ovulatory process. Hum Reprod 1991;6, 1206-1212.

34 Bordone, L. et al. SIRT1 transgenic mice show phenotypes resembling calorie restriction. Aging cell 2007;6, 759-767.

Table 1: Distribution Of The Descriptive Characteristics Of The Study Subjects 


\begin{tabular}{llll}
\hline Variables & Fertile $\mathrm{n}=207$ Mean $\pm \mathrm{SD}$ & Infertile $\mathrm{n}=135$ Mean $\pm \mathrm{SD}$ & $\mathrm{P}$ value \\
\hline Age (years) & $31.22 \pm 6.02$ & $31.13 \pm 5.7$ & 0.896 \\
Estradiol $(\mathrm{pg} / \mathrm{ml}$ & $230 \pm 5.37$ & $122.45 \pm 32.64$ & $<0.001$ \\
FSH (IU/L) & $5.67 \pm 2.38$ & $5.6 \pm 2.1$ & 0.780 \\
LH (IU/L) & $14.23 \pm 5.32$ & $6.72 \pm 3.49$ & $<0.001$ \\
Progesterone (nmol/L) & $66.28 \pm 64.28$ & $54.23 \pm 44.27$ & 0.041 \\
Manganese SOD (ng/ml) & $2.32 \pm 0.58$ & $0.89 \pm 0.8$ & $<0.001$ \\
SIRT1 $(\mathrm{ng} / \mathrm{ml})$ & $5.25 \pm 2.83$ & $1.82 \pm 1.93$ & $<0.001$ \\
\hline
\end{tabular}

Values are expressed in Mean \pm S.D. Groups are compared by Mann- Whitney U test; where $p<0.001$ is considered significant

Table 2: Genotype And Frequencies of SIRT1 SNPs (rs10509291 and rs12778366) In the study subjects

\begin{tabular}{llllll}
\hline Genotype & $\begin{array}{l}\text { Fertile }(\mathbf{n}= \\
\mathbf{2 0 7})\end{array}$ & $\begin{array}{l}\text { Infertile } \\
(\mathbf{n = 1 3 5})\end{array}$ & OR $(\mathbf{9 5 \%} \mathbf{C I})$ & OR $(\mathbf{9 5 \%}$ CI $)$ & P value \\
rs10509291 & rs10509291 & rs10509291 & rs10509291 & rs10509291 & rs10509291 \\
AA & $1(0 \%)$ & $10(7 \%)$ & $0.05(0.01-0.4)$ & $0.05(0.01-0.4)$ & 0.005 \\
TA & $16(8 \%)$ & $29(21 \%)$ & $0.28(0.14$ & $0.28(0.14$ & $<0.001$ \\
& & & $-0.54)$ & $-0.54)$ & \\
TT & $190(92 \%)$ & $96(71 \%)$ & Referent & Referent & - \\
rs12778366 & rs12778366 & rs12778366 & rs12778366 & rs12778366 & rs12778366 \\
CC & $1(0 \%)$ & $18(13 \%)$ & $0.02(0-0.19)$ & $0.02(0-0.19)$ & $<0.001$ \\
TC & $13(6 \%)$ & $31(23 \%)$ & $0.19(0.09$ & $0.19(0.09$ & $<0.001$ \\
& & & $-0.37)$ & $-0.37)$ & \\
TT & $193(93 \%)$ & $86(64 \%)$ & Referent & Referent & - \\
\hline
\end{tabular}

A p value of $<0.05$ considered significant. Genotype frequencies are expressed in the absolute values with percentages given in brackets.

Table 3: Association of Alleles of SIRT1 SNPs (rs12778366 and rs10509291) in all study subjects

\begin{tabular}{|c|c|c|c|c|c|}
\hline SNP Allele & $\begin{array}{l}\text { Fertile }(n= \\
207)\end{array}$ & $\begin{array}{l}\text { Infertile }(\mathrm{n}= \\
135)\end{array}$ & Chi Square & $\begin{array}{l}\text { Allele data OR } \\
(95 \% \text { CI })\end{array}$ & p-value \\
\hline rs10509291 & rs10509291 & rs10509291 & rs10509291 & rs10509291 & rs10509291 \\
\hline A & $18(4 \%)$ & $49(18 \%)$ & $\begin{array}{l}27.41 \\
(\mathrm{p}=<0.001)\end{array}$ & $0.12(0.05-0.3)$ & $<0.001$ \\
\hline $\mathbf{T}$ & $396(96 \%)$ & $221(82 \%)$ & & & \\
\hline rs12778366 & rs12778366 & rs12778366 & rs12778366 & rs12778366 & rs12778366 \\
\hline $\mathrm{C}$ & $15(4 \%)$ & $67(25 \%)$ & $\begin{array}{l}35.13 \\
(p=<0.001)\end{array}$ & $0.17(0.09-0.32))$ & $<0.001$ \\
\hline $\mathbf{T}$ & $399(96 \%)$ & $203(75 \%)$ & & & \\
\hline
\end{tabular}

The $\mathrm{p}$ value of $<0.05$ was considered significant and was calculated by Chi square test. $\mathrm{T}$ is the referent allele. Allele frequencies are given in absolute values with percentages given in parenthesis.

Table 4: Association of Genotype with Fertility. 


\begin{tabular}{lllll}
\hline \multirow{2}{*}{ Genotype } & Existence of SNP & Fertile $(\mathbf{n}=\mathbf{2 0 7})$ & $\begin{array}{l}\text { Infertile }(\mathbf{n}= \\
\mathbf{1 3 5})\end{array}$ & $\begin{array}{l}\text { Chi square P } \\
\text { value }\end{array}$ \\
\hline rs10509291 & $\mathrm{AA} / \mathrm{AT}$ & $17(8 \%)$ & $39(29 \%)$ & $<0.001$ \\
& $\mathrm{TT}$ & $190(92 \%)$ & $96(71 \%)$ & \\
rs12778366 & $\mathrm{CC} / \mathrm{CT}$ & $14(7 \%)$ & $49(36 \%)$ & $<0.001$ \\
& $\mathrm{TT}$ & $193(93 \%)$ & $86(64 \%)$ & \\
\hline
\end{tabular}

Values are expressed in $\mathrm{n}(\%)$. $\mathrm{p}$ value $<0.01$ is significant.

Table 5 (a): Comparison of study parameters between SNP rs12778366 (CC/CT) and TT females.

\begin{tabular}{llll}
\hline Variables & CT/CC $(\mathbf{n}=\mathbf{6 4})$ & TT $(\mathbf{n}=\mathbf{2 7 8})$ & $\mathbf{p}$ value \\
\hline & $1.15 \pm 1.16$ & $1.9 \pm 1.5$ & $<0.001$ \\
SIRT1 $(\mathrm{ng} / \mathrm{ml})$ & $2.5 \pm 2.4$ & $4.2 \pm 3.1$ & $<0.001$ \\
Estradiol $(\mathrm{pg} / \mathrm{ml})$ & $233.052 \pm 250.4$ & $194.591 \pm 248.6$ & 0.271 \\
Progesterone $(\mathrm{nmol} / \mathrm{L})$ & $71.40 \pm 56.46$ & $59.30 \pm 57.6$ & 0.130 \\
$\begin{array}{l}\text { Follicle Stimulating } \\
\text { (IU/L) }\end{array}$ & $6.31 \pm 2.5$ & $5.5 \pm 2.4$ & 0.018 \\
$\begin{array}{l}\text { Leutinizing Hormone } \\
\text { (IU/L) }\end{array}$ & $5.29 \pm 2.64$ & $9.151 \pm 4.5$ & $<0.001$ \\
\hline
\end{tabular}

Where MnSOD: Manganese/mitochondrial superoxide dismutase, The values are given in absolute values. $\mathrm{P}<0.05$ is considered significant.

(b) Comparison of study parameters between SNP rs10509291 (AA/AT) and TT females.

\begin{tabular}{llll}
\hline Variables & AA $/ \mathbf{A T}(\mathbf{n}=\mathbf{6 3})$ & TT $(\mathbf{n}=\mathbf{2 7 9})$ & $\mathbf{p}$ value \\
\hline MnSOD $(\mathrm{ng} / \mathrm{ml})$ & $1.3 \pm 1.25$ & $1.9 \pm 1.5$ & 0.02 \\
SIRT1 $(\mathrm{ng} / \mathrm{ml})$ & $2.1 \pm 0.2$ & $4.9 \pm 3.0$ & $<0.001$ \\
Estradiol $(\mathrm{pg} / \mathrm{ml})$ & $198.7 \pm 239.4$ & $231.12 \pm 252.3$ & 0.39 \\
Progesterone $(\mathrm{nmol} / \mathrm{L})$ & $60.28 \pm 56.15$ & $61.7 \pm 52.8$ & 0.86 \\
$\begin{array}{l}\text { Follicle Stimulating } \\
\text { (IU/L) }\end{array}$ & $5.5 \pm 2.4$ & $5.7 \pm 2.5$ & 0.70 \\
$\begin{array}{l}\text { Leutinizing Hormone } \\
\text { (IU/L) }\end{array}$ & $5.3 \pm 4.6$ & $7.3 \pm 4.5$ & $<0.001$ \\
\hline
\end{tabular}

Where MnSOD: Manganese/mitochondrial superoxide dismutase, The values are given in absolute values. $\mathrm{P}<0.05$ is considered significant.

\section{Additional Information:}

Conflict of interest:

The authors declare no conflict of interest.

Author Information:

Faiza Alam, Rehana Rehman and Taseer Ahmed Khan

They conceived and designed the experiments. 


\section{Mussarat Ashraf}

MA computed and analyzed data.

Faiza Alam, Rehana Rehman and Taseer Ahmed Khan, Mussarat Ashraf and Syeda Sadia Fatima

All contributed equally for manuscript writing. MA \& SSF prepared figure 1 while FA constructed figure 2. Funding:

Pakistan Science Foundation (PSF) grant \#PSF/Res/S-AKU/Med(479)

Figure 1 : Genotype: electrophoresis of amplified product of SIRT 1 (rs12778366 \& rs10509291 A, B), DNA electropherogram of SIRT 1 (rs12778366 \& rs10509291 C-H)

Gel electrophoresis and sequencing chromatograms of SNPs in SIRT1 gene. A \& B: PCR amplification shown as bands on $2 \%$ agarose gel of rs10509291 (372bp) and rs12778366 (911bp) of fertile and infertile samples numbered S1-S11 and S1-S6 respectively. C, D \& E: Sequencing chromatograms showing heterozygous and homozygous (AT, AA \& TT) rs10509291 SNPs and (F, G \& H) showing heterozygous and homozygous (CT, CC, TT) rs12837766 SNPs.

Figure 2: Hypothetical view of the possible mechanisms by which SIRT1 affects the microenvironment of the granulosa cells.

With advancing age of the oocytes, development of oxidative stress takes place which leads to imbalance between the oxidants and the antioxidants thus elevating reactive oxygen species, lipid peroxidation and simultaneously decreasing the expression of SIRT1 via decreased NAD/NADPH ratio (STEP1). These changes lead to mitochondrial dysfunction by direct injury to the mitochondrial DNA (STEP2) depleting ATP synthesis by the electron transport chain (STEP3). Stress causes release in cortisol due to the disturbed HPO and HPA axis. Oocyte maturation failure, chromosomal segregation disorders \& oocyte/embryo fragmentation occurs (STEP4) as a result causing infertility.
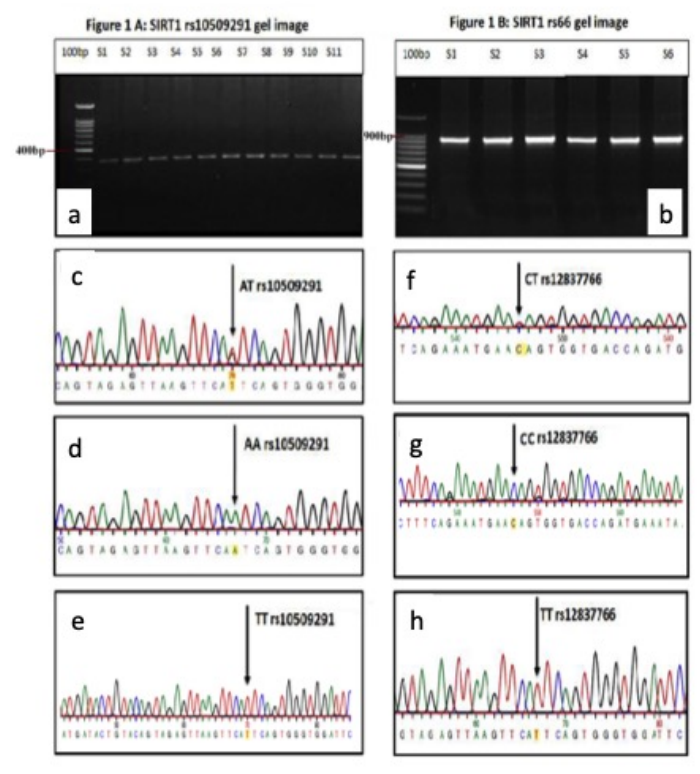


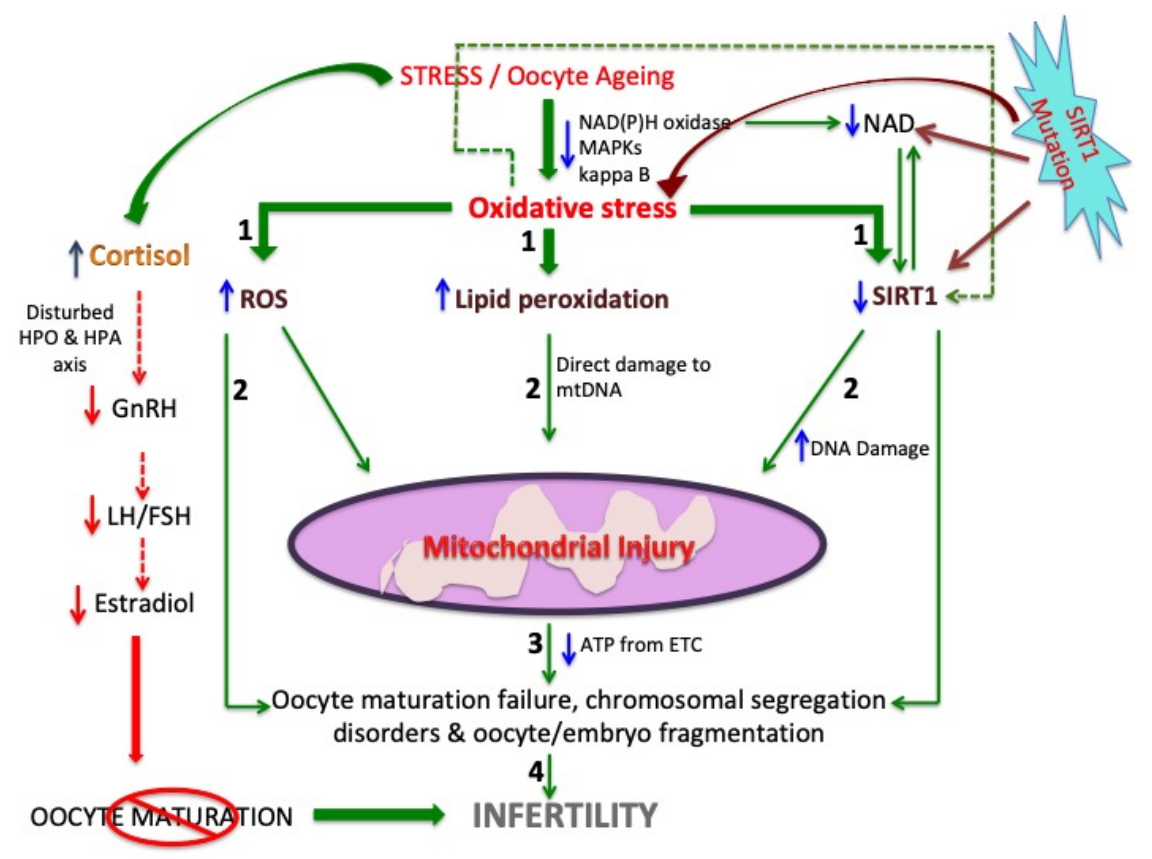

